\title{
A New Family of Non-Standard Finite Difference Schemes for the Logistic Equations
}

\author{
A.A. Obayomi and B. T. Olabode \\ Department of Mathematical Sciences, Ekiti State University, P. M.B 5363,Ado-Ekiti, \\ Ekiti-State, Nigeria. aaobayomi@yahoo.com \\ Department of Mathematical Sciences, Federal University of Technology, P.M.B 704 Akure, \\ Ondo-State, Nigeria. (+2348039739771) olabodebola@yahoo.com.
}

\begin{abstract}
This paper proposed a new family of Non-standard finite difference schemes for the Logistic equations. The technique of non-local approximation and renormalization of the denominator function was employed. The new schemes were found to possess desirable stability properties and also preserve all the monotonic properties of the logistic equations.
\end{abstract}

Keyword: Logistic Equation, Harvesting Model, Non-Standard Methods, Qualitative Stability.

\section{INTRODUCTION}

Analysis has been the most dominant part of mathematics and differential equation is the heart of analysis. A differential equation is the natural goal of elementary calculus and the most important part of mathematics for understanding the physical sciences.

However a lot of differential equations in the models, physical/real life situations does not possess analytic solutions or cannot be solved by a straight forward formula. This calls for numerical approximations. Numerical methods have gained more performance since the advent of computers. Softwares have been developed to simulate numerical experiment with expected real life situation.

Non-standard numerical methods were introduced by Mickens (1994) as a viable tool that provides approximate solution to differential equations and retain the qualitative properties of the equation. In Mickens (1994, 2000), valuable reasons for numerical instabilities were given in some particular investigated cases. The preservation of the qualitative properties of the considered differential equation with respect to these schemes is of great interest in finite difference methods of solving differential equations. The major consequence of this result is that such scheme does not allow numerical instabilities to occur. He proposed a new method of construction of discrete models whose solution have the same qualitative properties as that of the corresponding differential equations for all step-sizes and thus eliminate the elementary numerical instabilities that can arise.
The general form of the Logistic equation can be represented by the expression below:

$$
\frac{\partial y}{\partial x}=y(a-b y)+h
$$

Equation (1) can model population growth of a population dynamic and it is called logistic equation. The function $\mathrm{h}$ represents an external phenomenon that has direct impact on the population growth as applicable in immigration, emigration and fish farming for example. The function $\mathrm{h}$ may be time dependent or population dependent. In fish farming for example, if $h(y) \neq 0$ then it is called a harvesting or restocking model where $h(y)$ is the rate of harvesting or restocking. If $h(y)>0$ and is a constant then it is called a constant restocking model.

If $h(y)=\propto y, \propto$ is the proportion of harvesting/restocking in a period in time then,

$$
y^{\prime}=y(a-b y)+\propto y .
$$

Harvesting when $\propto<0$ and restocking when $\propto>0$. The term $\propto y$ is the proportional harvesting model while $-b y^{2}$ is referred to as the inhibition or competition term and $\propto y$ is the reproduction / natural growth rate model of the specie.

However, if we consider the population dynamics of a fairly exclusive eco-system i.e induced and external impacts like harvesting, restocking etc are not allowed or inexistence then the general form of logistic equation can be represented in the form:

$$
y^{\prime}=\alpha_{1} y-\alpha_{2} y^{2}
$$

whose dimensionless form is 


$$
y^{\prime}=y-y^{2}
$$

This means that all non-essential constants and parameters that arise in the original differential equations have been eliminated (see Mickens (1994)). Note that the "physical" original differential equation connects the derivatives of a physical variable such as distance or current and its relations to the various physical parameters, while the transformed dimensionless equation relates the various derivatives of a "mathematical" variable and associated constant that appear in the equation. For the purpose of the numerical experiment, we shall consider the dimensionless, non-dimensionless and harvesting model of the logistic equation.

The initial value problem of the dimensionless model of the logistic equation is given by

$$
\mathrm{y}^{\prime}=y(1-y) \quad \text { or } y-y^{2}, \quad y(0)=y_{0} .
$$

This is a non-linear homogeneous first order differential equation with a general solution of the form:

$$
y(t)=\frac{y_{0} e t}{y_{0}+y_{0} e^{t}}
$$

This equation has two equilibrium points 0 and 1 . The equilibrium point 1 is stable while the point 0 is unstable. The table below shows the monotonic property of the Logistic equation:

\begin{tabular}{|c|c|c|c|}
\hline $\begin{array}{l}\text { Qualitative } \\
\text { Property of } \\
\text { the IVP }\end{array}$ & $\begin{array}{l}\text { Decreases to }-\infty \text { at } \\
\text { the singular point } \\
t=t^{*}=\operatorname{Ln}\left[\frac{1+\left|y_{0}\right|}{\left|y_{0}\right|}\right] \text {, } \\
\text { after which, for } \\
t>t^{*} \text {, it assume a } \\
\text { positive value then } \\
\text { decreases } \\
\text { monotonically to the } \\
\text { fixed-point at } \\
y(t)=1 \text {. }\end{array}$ & $\begin{array}{l}\text { Increasing } \\
\text { with limit } 1\end{array}$ & $\begin{array}{l}\text { Decrea } \\
\text { sing } \\
\text { with } \\
\text { limit } 1\end{array}$ \\
\hline $\begin{array}{l}\text { Interval of } \\
\text { Initial Value }\end{array}$ & $(-\infty, 0)$ & $(0,1)$ & $(1, \infty)$ \\
\hline
\end{tabular}

Table 1: Monotonic Properties of the Logistic Equation

This paper therefore proposes non-standard finite difference schemes that preserve the above qualitative properties.

Some New Qualitatively Stable Non-Standard Finite Difference Schemes (NSFDS): The following Non-standard discretization and denominator functions for the dimensionless logistic equation had earlier been considered by Ibijola and Obayomi (2012) for the dimensionless equation and used for initial values close to the fixed points ( 0 and 1$)$. The said scheme was also considered by Anguelov and Lubuma (2003) for analysis of P-stable Finite difference schemes but the renormalized denominator functions were not considered. In this work we will be considering higher initial values and longer interval of integration and positive solution only. We have also introduced new denominator functions that satisfy the rule 2 of the non-standard modeling rules (see Mickens (1994)).

We shall consider the Non-standard finite difference scheme for any dimensionless equation

$y^{\prime}=f(x, y)$ given by

$$
f\left(x_{n}, y_{n}\right) \quad \text { or } \quad y_{n+1}+\varphi f\left(x_{n}, y_{n}\right) \text { (7) }
$$

Hence, we have Non-standard schemes

Scheme S1 $y_{n+1}=\frac{y_{n}+\varphi y_{n}-(1-a) \varphi y_{n}^{2}}{1+a \varphi y_{n}}, \varphi=\left[\frac{\left(e^{\lambda h}-1\right)}{\lambda}\right]$
Scheme S2 $y_{n+1}=\frac{y_{n}+\varphi y_{n}-(1-a) \varphi y_{n}^{2}}{1+a \varphi y_{n}}, \varphi=h\left[\frac{n h+\delta)}{n h+h+\delta}\right]$
Scheme S3 $y_{n+1}=\frac{y_{n}+\varphi y_{n}-(1-a) \varphi y_{n}^{2}}{1+a \varphi y_{n}}, \varphi=\mathrm{h}$
Scheme S4 $y_{n+1}=\frac{y_{n}+\varphi y_{n}-(1-a) \varphi y_{n}^{2}}{1+a \varphi y_{n}}, \varphi=\left(h+h^{2}+\frac{h^{2}}{2 n^{3}}\right)$

Scheme S5 $y_{n+1}=\frac{y_{n}+\varphi y_{n}-a \varphi y_{n}^{2}}{1+(1-a) \varphi y_{n}}, \varphi=\left[\frac{\left(e^{\lambda h}-1\right)}{\lambda}\right]$

Scheme S6 $y_{n+1}=\frac{y_{n}+\varphi y_{n}-a \varphi y_{n}^{2}}{1+(1-a) \varphi y_{n}}, \varphi=h\left[\frac{n h+\delta)}{n h+h+\delta}\right]$

Scheme S7 $y_{n+1}=\frac{y_{n}+\varphi y_{n}-a \varphi y_{n}^{2}}{1+(1-a) \varphi y_{n}}, \varphi=\mathrm{h}$

Scheme S8 $y_{n+1}=\frac{y_{n}+\varphi y_{n}-a \varphi y_{n}^{2}}{1+(1-a) \varphi y_{n}}, \varphi=\left(h+h^{2}+\frac{h^{2}}{2 n^{3}}\right)$

New NSFDS for the harvesting model of the logistic equation.

The derivation of the discretization functions are discussed here. From the initial value problem

$$
y^{\prime}=\mathrm{A} y-\mathrm{B} y^{2}-\mathrm{C}, y(0)=y_{0} \text {. }
$$

Consider the following convex approximation to the non-linear term $y^{2}$ as given below

$$
y^{2}=a y_{n} y_{n+1}+(1-a) y_{n}^{2}
$$

Using (7), (16) and (17), we have $\frac{y_{n+1}-y_{n}}{\varphi}=\mathrm{A} y_{n}-$ $\mathrm{B}(1-a) y_{n}^{2}-a B y_{n} y_{n+1}-\mathrm{C}$

a) $\varphi y_{n}^{2}-\varphi C$

$$
\Rightarrow\left(1+a \varphi B y_{n}\right) y_{n+1}=y_{n}+\varphi A y_{n}-\mathrm{B}(1-
$$

$$
\Rightarrow y_{n+1}=\frac{y_{n}+\varphi A y_{n}-\mathrm{B}(1-a) \varphi y_{n-\varphi C}^{2}}{\left(1+a \varphi B y_{n}\right)}
$$


Note that if we apply a nonlocal approximation of $y^{2}=a y_{n}^{2}+(1-a) y_{n} y_{n+1}$

Then, the scheme becomes

$$
y_{n+1}=\frac{y_{n}+\varphi A y_{n}-a \varphi y_{n-\varphi C}^{2}}{\left(1+(1-a) \varphi B y_{n}\right)}
$$

Applying

$\varphi=h\left(\frac{n h+\delta}{(n+1) h+\delta}\right), h,\left(h+h^{2+}+\frac{h^{2}}{2 n^{3}}\right),\left[\frac{\left(e^{\lambda h}-1\right)}{\lambda}\right]$

yields the new schemes. It can be shown that all denominator functions in the equation no 22 obeys the Micken (1994)'s rule 2 i.e $\varphi(h)=h+O\left(h^{2}\right)$ as $h$ $\rightarrow 0$.

Numerical Examples and Experiments: In this section, we present the numerical examples and experiments.

\section{Example 1}

Example of non-dimensionless Logistic equation

$$
y^{\prime}=f(x, y)=y(1-0.0005 y), y(0)=1
$$

The analytic solution is $y=\frac{2000 e^{x}}{1999+e^{x}}$

Using the renormalized denominator functions in equation (23) and setting $A=1, B=0.0005, \quad C=0$, one obtains the following schemes.

Scheme C1 $y_{n+1}=\frac{y_{n}\left(1+\varphi-0.0005 \varphi \mathrm{a} y_{n}\right)}{\left(1+0.0005 \mathrm{~b} \varphi \mathrm{y}_{\mathrm{n}}\right)}$,

$\mathrm{a}+\mathrm{b}=1, \varphi=\left(\frac{\left(e^{\lambda \boldsymbol{h}}-1\right)}{\lambda}\right)$

Scheme C2 $y_{n+1}=\frac{y_{n}\left(1+\varphi-0.0005 \varphi \mathrm{a} y_{n}\right)}{\left(1+0.0005 \mathrm{~b} \varphi y_{n} \varphi \mathrm{y}_{\mathrm{n}}\right)}$ $\mathrm{a}+\mathrm{b}=1, \varphi=h\left[\frac{n h+400}{n h+h+400}\right]$

Scheme C3 $y_{n+1}=\frac{y_{n}\left(1+\varphi-0.0005 \varphi \mathrm{a} \quad y_{n}\right)}{\left(1+0.0005 \mathrm{~b} \varphi \mathrm{y}_{\mathrm{n}}\right)}$,

$\mathrm{a}+\mathrm{b}=1, \varphi=h$

Scheme C4 $y_{n+1}=\frac{y_{n}\left(1+\varphi-0.0005 \varphi \mathrm{a} y_{n}\right)}{\left(1+0.0005 \mathrm{~b} \varphi \mathrm{y}_{\mathrm{n}}\right)}$,

$\mathrm{a}+\mathrm{b}=1, \varphi=\left(h+h^{2+}+\frac{h^{2}}{2 n^{3}}\right)$

\section{Example 2}

Consider the harvesting model

$$
y^{\prime}=5 y-y^{2}-4, \quad y(0)=y_{o}
$$

With analytic solution $y_{(t)}=\frac{4\left(y_{o}-1\right)-\left(y_{o}-4\right) e^{-(3 t)}}{\left(y_{o}-1\right)-\left(y_{o}-4\right) e^{-(3 t)}}$ Using (21) and (22), one obtains the following schemes

Scheme D1 $y_{n+1}=\frac{y_{n}+5 \varphi y_{n}-(1-a) \varphi y_{n}^{2}-4 \varphi}{\left(1+a \varphi y_{n}\right)}$,

$\varphi=\left[\frac{\left(e^{\lambda \boldsymbol{h}}-1\right)}{\lambda}\right]$

Scheme D2 $y_{n+1}=\frac{y_{n}+5 \varphi y_{n}-(1-a) \varphi y_{n}^{2}-4 \varphi}{\left(1+a \varphi y_{n}\right)}$,

$\varphi=\left[\frac{\left(e^{\lambda \boldsymbol{h}}-1\right)}{\lambda}\right]$

Scheme D3 $y_{n+1}=\frac{y_{n}+5 \varphi y_{n}-(1-a) \varphi y_{n}^{2}-4 \varphi}{\left(1+a \varphi y_{n}\right)}, \varphi=\mathrm{h}$

Scheme D4 $y_{n+1}=\frac{y_{n}+5 \varphi y_{n}-(1-a) \varphi y_{n}^{2}-4 \varphi}{\left(1+a \varphi y_{n}\right)}$,

$\varphi=\left(h+h^{2+}+\frac{h^{2}}{2 n^{3}}\right)$

Table 2 : The schemes for the Dimensionless Logistic Equations

\begin{tabular}{|c|l|l|}
\hline 1 & $y_{(t)}=\frac{y_{o}}{y_{o}+\left(1-y_{o}\right) e^{-(t)}}$ & $\begin{array}{l}\text { Analytic Scheme for the dimensionless } \\
\text { Logistic equation }\end{array}$ \\
\hline 2 & $y_{n+1}=\frac{y_{n}+\varphi y_{n}-(1-a) \varphi y_{n}^{2}}{1+a \varphi y_{n}}, \varphi=\left[\frac{\left(e^{\lambda h}-1\right)}{\lambda}\right]$ & Scheme S1 \\
\hline 3 & $y_{n+1}=\frac{y_{n}+\varphi y_{n}-(1-a) \varphi y_{n}^{2}}{1+a \varphi y_{n}}, h\left[\frac{n h+400}{n h+h+400}\right]$ & Scheme S2 \\
\hline 4 & $y_{n+1}=\frac{y_{n}+\varphi y_{n}-(1-a) \varphi y_{n}^{2}}{1+a \varphi y_{n}}, \varphi=\mathrm{h}$ & Scheme S3 \\
\hline 5 & $y_{n+1}=\frac{y_{n}+\varphi y_{n}-(1-a) \varphi y_{n}^{2}}{1+a \varphi y_{n}}, \varphi=\left(h+h^{2}+\frac{h^{2}}{2 n^{3}}\right)$ & Scheme S4 \\
\hline 6 & $y_{n+1}=\frac{y_{n}+\varphi y_{n}-a \varphi y_{n}^{2}}{1+(1-a) \varphi y_{n}}, \varphi=\frac{\left(e^{\lambda h}-1\right)}{\lambda}$ & Scheme S5 \\
\hline 7 & $y_{n+1}=\frac{y_{n}+\varphi y_{n}-a \varphi y_{n}^{2}}{1+(1-a) \varphi y_{n}}, \varphi=h\left[\frac{n h+400}{n h+h+400}\right]$ & Scheme S6 \\
\hline 8 & $y_{n+1}=\frac{y_{n}+\varphi y_{n}-a \varphi y_{n}^{2}}{1+(1-a) \varphi y_{n}}, \varphi=h$ & Scheme S7 \\
\hline 9 & $y_{n+1}=\frac{y_{n}+\varphi y_{n}-a \varphi y_{n}^{2}}{1+(1-a) \varphi y_{n}} \varphi=\left(h+h^{2}+\frac{h^{2}}{2 n^{3}}\right)$ & Scheme S8 \\
\hline
\end{tabular}


Table 3 : The new schemes for the non-dimensionless Logistic equations.

\begin{tabular}{|l|c|l|}
\hline 1 & $y=\frac{2000 e^{k h}}{1999+e^{k h}}$ & $\begin{array}{l}\text { Analytic solution for } \\
y^{\prime}=y(1-0.0005 y), y(0)=1\end{array}$ \\
\hline 2 & $y_{n+1}=\frac{y_{n}\left(1+\varphi-0.0005 \varphi \mathrm{a} y_{n}\right)}{\left(1+0.0005 \mathrm{~b} \varphi \mathrm{y}_{\mathrm{n}}\right)}, \varphi=\left(\frac{\left(e^{\lambda h}-1\right)}{\lambda}\right)$ & $\mathrm{C} 1$ \\
\hline 3 & $y_{n+1}=\frac{y_{n}\left(1+\varphi-0.0005 \varphi \mathrm{a} y_{n}\right)}{\left(1+0.0005 \mathrm{~b} \varphi \mathrm{y}_{\mathrm{n}}\right)}, \varphi=h\left[\frac{n h+400}{n h+h+400}\right]$ & $\mathrm{C} 2$ \\
\hline 4 & $y_{n+1}=\frac{y_{n}\left(1+\varphi-0.0005 \varphi \mathrm{a} y_{n}\right)}{\left(1+0.0005 \mathrm{~b} \varphi \mathrm{y}_{\mathrm{n}}\right)}, \varphi=h$ & $\mathrm{C} 3$ \\
\hline 5 & $y_{n+1}=\frac{y_{n}\left(1+\varphi-0.0005 \varphi \mathrm{a} y_{n}\right)}{\left(1+0.0005 \mathrm{~b} \varphi \mathrm{y}_{\mathrm{n}}\right)}, \varphi=\left(h+h^{2+}+\frac{h^{2}}{2 n^{3}}\right)$ & $\mathrm{C} 4$ \\
\hline 6 & $y_{n+1}=\frac{y_{n}+\varphi y_{n}-0.0005(1-a) \varphi y_{n}^{2}}{\left(1+0.0005 a \varphi y_{n}\right)}, \varphi=\frac{\left(e^{\lambda h}-1\right)}{\lambda}$ & $\mathrm{C} 5$ \\
\hline
\end{tabular}

Table 4 : The new schemes for a harvesting model of the Logistic equation.

\begin{tabular}{|c|l|c|}
\hline 1 & $y_{(t)}=\frac{4\left(y_{o}-1\right)-\left(y_{o}-4\right) e^{-(3 t)}}{\left(y_{o}-1\right)-\left(y_{o}-4\right) e^{-(3 t)}}$ & $\begin{array}{c}\text { Analytic solution for the model } \\
y^{\prime}=5 y-y^{2}-4, y(0)=y_{o}\end{array}$ \\
\hline 2 & $y_{n+1}=\frac{y_{n}+\varphi A y_{n}-\mathrm{B}(a) \varphi y_{n-\varphi C}^{2}}{\left(1+(1-\mathrm{a}) a \varphi B y_{n}\right)}, \varphi=\frac{\left(e^{\lambda h}-1\right)}{\lambda}$ & $\mathrm{D} 1$ \\
\hline 3 & $y_{n+1}=\frac{y_{n}+\varphi A y_{n}-\mathrm{B}(a) \varphi y_{n-\varphi C}^{2}}{\left(1+(1-\mathrm{a}) a \varphi B y_{n}\right)}, \varphi=h\left[\frac{n h+\delta}{n h+h+\delta}\right]$ & $\mathrm{D} 2$ \\
\hline 4 & $y_{n+1}=\frac{y_{n}+\varphi A y_{n}-\mathrm{B}(a) \varphi y_{n-\varphi C}^{2}}{\left(1+(1-\mathrm{a}) a \varphi B y_{n}\right)}, \varphi=\mathrm{h}$ & $\mathrm{D} 3$ \\
\hline 5 & $y_{n+1}=\frac{y_{n}+\varphi A y_{n}-\mathrm{B}(a) \varphi y_{n-\varphi C}^{2}}{\left(1+(1-\mathrm{a}) a \varphi B y_{n}\right)}, \varphi=\left(h+h^{2}+\frac{h^{2}}{2 n^{3}}\right)$ & $\mathrm{D} 4$ \\
\hline 6 & $y_{n+1}=\frac{y_{n}+\varphi A y_{n}-\mathrm{B}(1-a) \varphi y_{n-\varphi C}^{2}}{\left(1+a \varphi B y_{n}\right)}, \varphi=\frac{\left(e^{\lambda h}-1\right)}{\lambda}$ & $\mathrm{D} 5$ \\
\hline
\end{tabular}

Stability Properties of New Non-standard Finite Difference Schemes: In this section, we shall adopt Anguelov and Lubuma (2003)'s idea in analyzing the qualitative property of the new Non-standard schemes. Consider an equation of the form:

$$
\mathrm{y}^{\prime}=\mathrm{f}(\mathrm{t}, \mathrm{y}), \mathrm{y}\left(\mathrm{t}_{0}\right)=\mathrm{y}_{0}
$$

Let us denote $y_{k}$ the approximate solution of (35) at grid point $t_{k}: y_{k} y\left(t_{k}\right)$, then the sequence $y_{k}$ is obtained a solution to a finite difference equation of the form:

$$
y_{k+1}=y_{k}+\mathrm{h} \varphi\left(x_{k}, y_{k} ; \mathrm{h}\right)
$$

Denoting (36) by the sequence

$$
y_{k}=\mathrm{F}\left(\mathrm{h} ; y_{k}\right)
$$

Definition 1 : (Anguelov and Lubuma(2003)).

Assume that the solution of equation (35) satisfy some property $\mathcal{P}$, then the numerical scheme (37) is called qualitatively stable with respect to property $\mathcal{P}$ or $\mathcal{P}$-stable, if for every value $h>0$ the set of solutions of (37) satisfies $\mathcal{P}$.

Definition 2: (Anguelov and Lubuma (2003))

A set $G(\Omega)$ of real-valued functions defined on a subset $\Omega$ of $\left[\mathrm{t}_{0}, \infty\right)$ monotonically depend on the initial value at $t_{0}$ if for every two functions $y, z \in G(\Omega)$ we have $\mathrm{y}\left(\mathrm{t}_{0}\right) \leq \mathrm{z}\left(\mathrm{t}_{0}\right) \Rightarrow \mathrm{y}(\mathrm{t}) \leq \mathrm{z}(\mathrm{t}), \mathrm{t} \in \Omega$.

Definition 3: (Anguelov and Lubuma (2003))

The finite difference scheme (37) is stable with respect to the property of monotonicity of solutions if for every $y_{0} \in \mathbb{R}$ the solution $y_{k}$ of (37) is an increasing or a decreasing sequence according as the $y(t)$ of equation (35) is increasing or decreasing.

Definition 4: (Anguelov and Lubuma (2003))

The finite difference method (37) is called elementary stable if for any value of the step size $h$, its only fixed points $\bar{y}$ are those of the differential equation (35), the linear stability property of each $\bar{y}$ being the same for 
both the differential equation and the discrete method.

The following theorems establish the conditions (sufficient) for the stability properties of the discrete equation (37). Proves of the theorems can be found in Anguelov and Lubuma (2003). The authors have been able to prove the condition for stability of the fixed points and link the properties of linear stability to elementary stability of the fixed points.

Theorem 1 : (Anguelov and Lubuma(2003)).

The difference scheme (37) is stable with respect to monotone dependence on initial value if

$$
\frac{\partial F}{\partial y}(h ; y) \geq 0, y \in \mathbb{R}, h>0
$$

Theorem 2 : (Anguelov and Lubuma(2003)).
Assume that the difference scheme (37) is stable with respect to monotone dependence on initial value. Assume also that for every $h>0$ the equations

$$
y=F(h ; y) \text { and } f(y)=0
$$

in y have the same roots considered their multiplicity. Then the difference scheme (37) is stable with respect to monotonicity of solutions.

Theorem 3 : (Anguelov and Lubuma(2003)).

Under the assumptions of theorem 2, the difference scheme (37) is elementary stable.

In this research work we shall apply theorem 1-3 to determine and use the parameters for which the nonstandard schemes developed for the logistic equations are stable

\section{Graphical representation of the solution obtained from the schemes}



Fig 1 : Family of the dimensionless Logistic scheme $y_{n+1}=\frac{y_{n}+\varphi y_{n}-(1-a) \varphi y_{n}^{2}}{1+a \varphi y_{n}}$ using parameters where the schemes are stable.



Fig 2: Error of approximation (deviation from the analytic solution) 
Am. J. Sci. Ind. Res., 2013, 4(3): 277-284



Fig 3 : Family of the Logistic scheme $y_{n+1}=\frac{y_{n}+\varphi y_{n}-a \varphi y_{n}^{2}}{1+(1-a) \varphi y_{n}}$

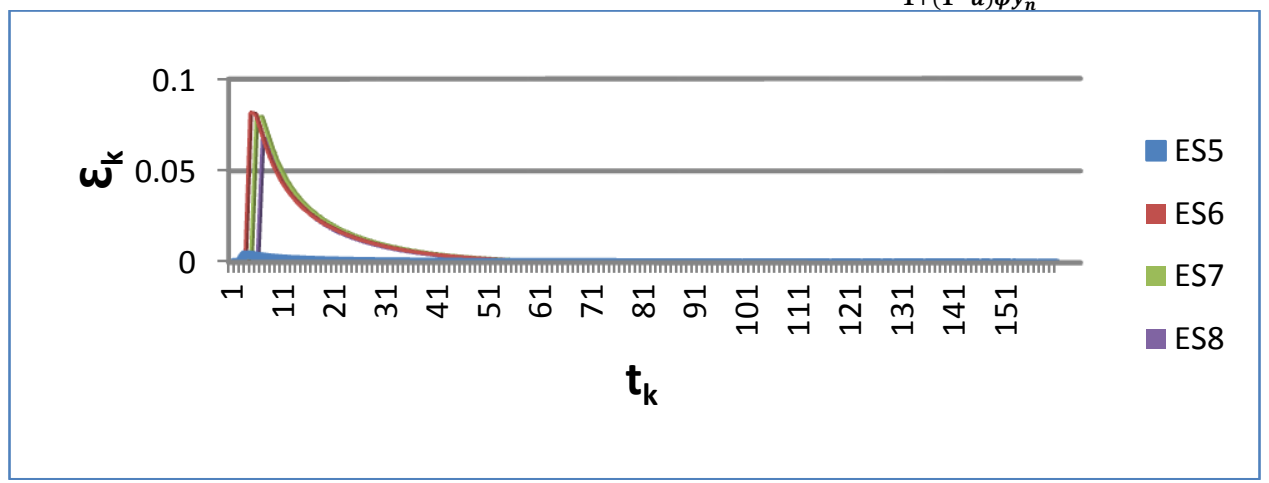

Fig 4 : Error of approximation(deviation from the analytic solution).



Fig 5 : Family of the Logistic scheme $y_{n+1}=\frac{y_{n}\left(1+\varphi-0.0005 \varphi \mathrm{a} y_{n}\right)}{\left(1+0.0005 \mathrm{~b} \varphi \mathrm{y}_{\mathrm{n}}\right)} \quad$. 
Am. J. Sci. Ind. Res., 2013, 4(3): 277-284

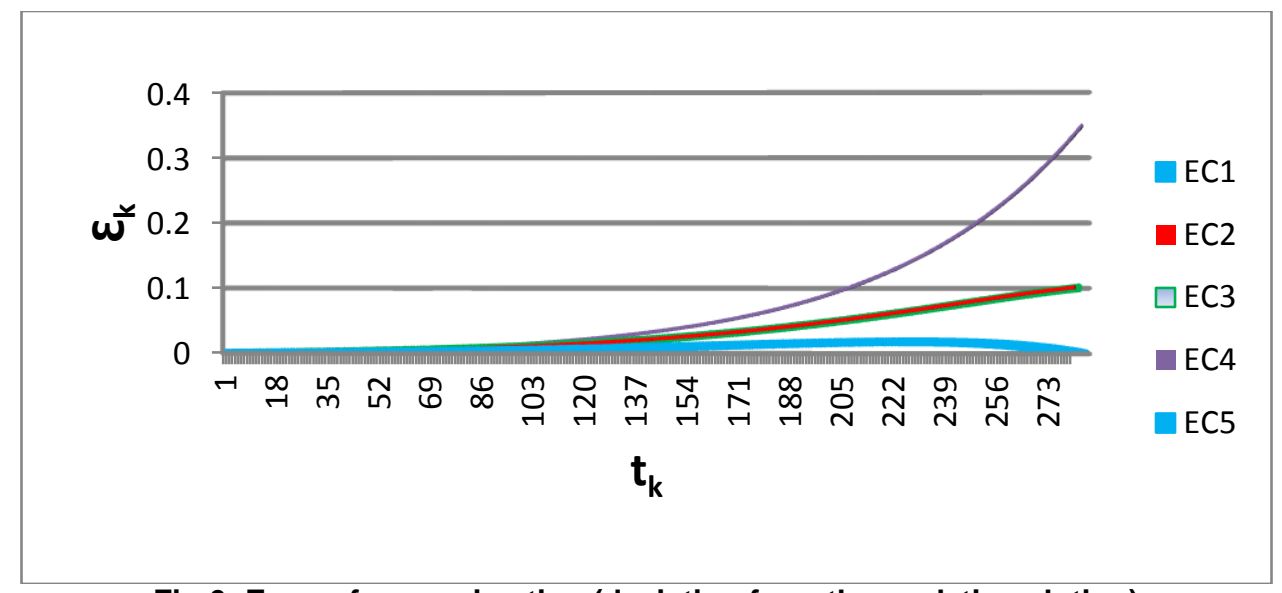

Fig 6: Error of approximation (deviation from the analytic solution)

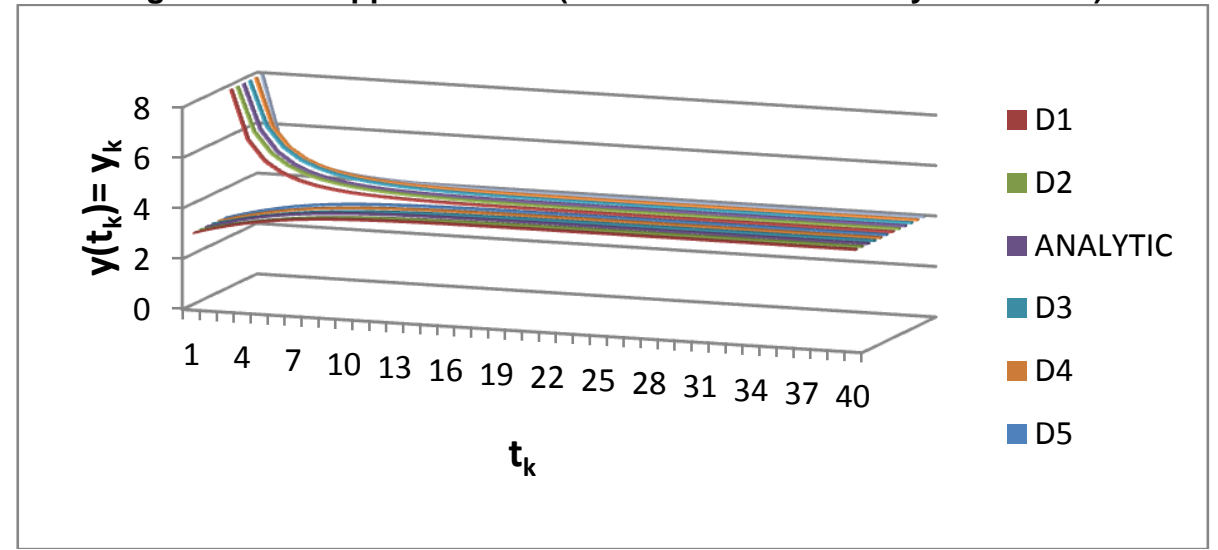

Fig 7: Family of the scheme $y_{n+1}=\frac{y_{n}+\varphi A y_{n}-\mathrm{B}(a) \varphi y_{n-\varphi C}^{2}}{\left(1+(1-\mathrm{a}) a \varphi B y_{n}\right)}$



Fig 8: Error of approximation (deviation from the analytic solution) below the equilibrium point 
Am. J. Sci. Ind. Res., 2013, 4(3): 277-284

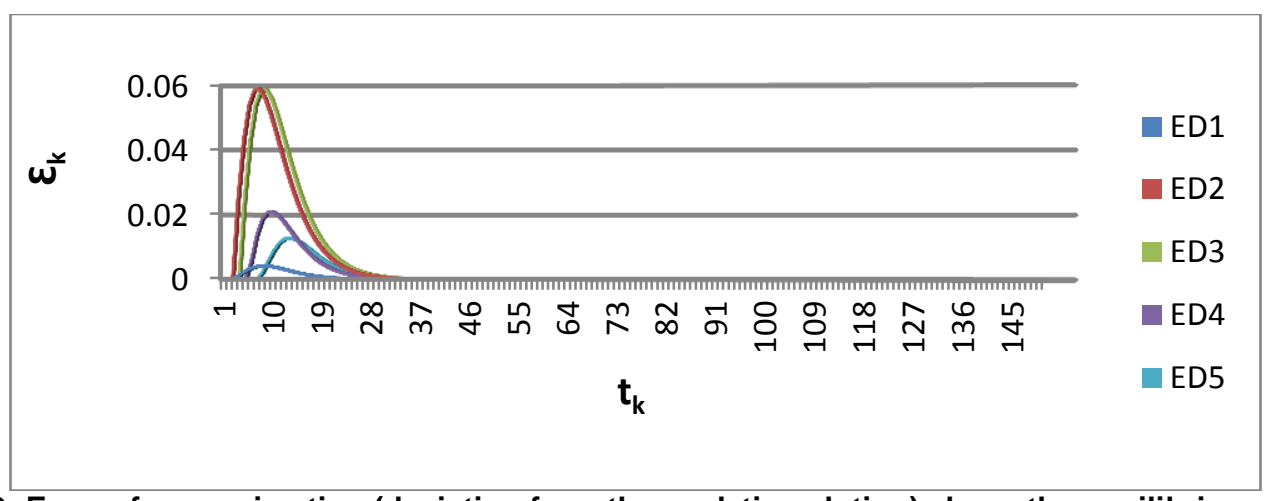

Fig 9: Error of approximation (deviation from the analytic solution) above the equilibrium point

\section{DISCUSSION OF RESULTS}

It will be observed that the solution curves of the new schemes did not intersect.

This confirms the three stability properties of (elementary stability, stability with respect to monotonicity of solution and stability with respect to monotone dependence on initial value). We have chosen the parameters $a, b$, and $\lambda$ for which the corresponding schemes become qualitatively stable after a series of numerical experiments. We particularly used Anguelov and Lubuma (2003)'s theorem on qualitative stability and test other various values of these parameters.

It must be noted that the error of approximation depends more on these parameters, there is no particularly best way of choosing these parameters, the choices becomes an art and it can be made in such a way that they can be better than each other depending on experience and familiarity with the equation under consideration. For example we have found out that the best renormalized denominator function for logistic schemes is $\varphi=\frac{\left(e^{\lambda h}-1\right)}{\lambda}$ from our experience. Here we can select our parameters carefully enough to have a qualitatively stable scheme and also the least error of approximation (see figures 1-9 for ES1, ES5, EC1, EC5, ED1, ED5). However, it is useful to note here that it is sufficient to select these parameters based on the theorem of Anguelov and Lubuma (2003) to obtain schemes that possess the three stability properties. In this work, we are interested in schemes that preserve the qualitative properties of the original equation.

\section{REFERENCES}

[1]. A. A. Obayomi (2012). A new set of Non-standard Finite Difference Schemes the solution of an Equation of the Type $y^{\prime}=y\left(1-y^{\mathrm{n}}\right)$. International Journal of
Pure and Applied Sciences and Technology12(2): 34 -42 .

[2]. D.G. Zill and R.M. Cullen (2005) Differential Equations with boundary value problems (sixth Edition) Brooks.

[3]. E.A. Ibijola and A.A. Obayomi (2012) A New Family Of Numerical Schemes for Solving Equation of the Type $y^{\prime}=y^{n}(1-y)$. Innova Sciencia vol 4 (6) June 2012 pages $34-46$

[4]. E.A. Ibijola and A.A. Obayomi (2012) A Numerical Scheme for the Solution of the Logistic Equation. Advances in Differential Equation and Control Processes Vol. 10 (1) pages 1- 18

[5]. J.M. Sanz-Serna(1985) SIAM Journal of Scientific and Statistical Computing 6, 923-938. Studies in Numerical nonlinear instability. Why do leap frog schemes go unstable?

[6]. M.Yamaguti $M$ and S. Ushiki (1981), Chaos in Numerical analysis of Ordinary Differential Equations. Physical 3D 618,626.

[7]. R. Anguelov, J.M.S. Lubuma (2000). Nonstandard finite difference method, by Non-local Approximation Keynote address at the South Africa Mathematical Society, Pretoria, South Africa 16-18 October, Notices of S. A. Math. Soc. 31: 143-543.

[8]. R. Anguelov, J.M.S. Lubuma (2003). Nonstandard finite difference method by nonlocal approximation, Mathematics and Computers in simulation 6: 465-475.

[9]. R.E. Mickens (1994). Nonstandard Finite Difference Models of Differential Equations, World Scientific, Singapore. Pp 1-115, 144-162.

[10].R. E. Mickens (2000). Applications of Nonstandard Methods for initial value problems, World Scientific, Singapore.

[11]. S. Ushiki (1982) Physical 1D, 407-424, Central Difference Scheme Chaos. 